\title{
Inhaled Glucocorticoid with or without Tiotropium Bromide for Asthma-Chronic Obstructive Pulmonary Disease Overlap Syndrome
}

\author{
Hailing $\mathrm{Xu}^{1}$ and Xiaoxia $\mathrm{Lu}^{2}$
}

\begin{abstract}
Objective: To compare the efficacy of inhaled glucocorticoid with or without tiotropium bromide in the treatment of patients with asthma-chronic obstructive pulmonary disease overlap syndrome (ACOS).

Study Design: An experimental study.

Place and Duration of Study: Department of Respiratory Medicine, Wuwei People's Hospital, Gansu Province, China, from October 2016 to October 2017.

Methodology: A total of 86 ACOS patients were randomly divided into the control group and the observation group, with 43 cases in each group. Control group was given inhaled glucocorticoid. Observation group was treated with tiotropium bromide on the basis of the control group. The asthma control test (ACT) score, chronic obstructive pulmonary disease assessment test (CAT) score, serum high-sensitivity C-reactive protein (hs-CRP) and IL-6 levels were compared.

Results: Before treatment, there was no significant difference in ACT score, CAT score, serum hs-CRP and IL-6 levels between the two groups ( $p=0.808,0.612,0.872$ and 0.921 , respectively). After treatment, ACT score in observation group was higher than that in control group $(\mathrm{p}<0.001)$. CAT score, serum hs-CRP, and IL-6 levels in observation group were lower than those in control group (all $p<0.001$ ). The incidence of adverse reactions was lower in observation group than that in control group $(\mathrm{p}=0.033)$.

Conclusion: Compared with inhaled glucocorticoid, inhaled glucocorticoid combined with tiotropium bromide treatment can more effectively reduce the serum levels of hs-CRP and IL-6 and is beneficial to control the development of ACOS.
\end{abstract}

Key Words: Asthma-chronic obstructive pulmonary disease overlap syndrome (ACOS), Glucocorticoid, Tiotropium bromide, Treatment, Efficacy.

\section{INTRODUCTION}

Asthma and chronic obstructive pulmonary disease (COPD) are the most common respiratory diseases in the clinic. Patients having bronchial obstruction due to inflammation in the airways, often present with clinical symptoms such as dyspnea, respiratory restriction, and cough.1,2 Related studies have shown that there are certain overlaps in the pathogenesis, clinical symptoms, and predisposing factors of asthma and chronic obstructive pulmonary disease. ${ }^{3}$ This overlap is referred to as asthma-chronic obstructive pulmonary overlap syndrome (ACOS). ${ }^{4}$ At present, many medicines are mainly used for the treatment of ACOS clinically, but there is no consensus as for which medicine to use. Glucocorticoid inhalation is a common method for the treatment of ACOS. Although it can improve the patient's lung function, the efficacy is not very satisfactory for

Department of Respiratory Medicine ${ }^{1}$ / Digestive2, Wuwei

People's Hospital, North of Xuanwu Street, Xincheng District, Wuwei City, Gansu Province, 733000, China

Correspondence: Xiaoxia Lu, Department of Digestive, Wuwei People's Hospital, North of Xuanwu Street, Xincheng District, Wuwei City, Gansu Province, 733000, China

E-mail:kcrk92@163.com

Received: June 26, 2018; Accepted: November 12, 2018 most patients. 5 Therefore, actively exploring effective treatment plan for ACOS is one of the urgent medical problems clinically. Tiotropium bromide is a bronchodilator suitable for the maintenance treatment of chronic obstructive pulmonary disease. 6

The objective of this study was to compare the efficacy of inhaled glucocorticoid with or without tiotropium bromide in the treatment of patients with asthma-chronic obstructive pulmonary disease overlap syndrome (ACOS).

\section{METHODOLOGY}

This experimental study was conducted at the Department of Respiratory Medicine, Wuwei People's Hospital, North of Xuanwu Street, Xincheng District, Wuwei City, Gansu Province, China, from October 2016 to October 2017. The study was approved by the Hospital Ethical and Research Committee. A total of 86 ACOS patients were selected as the study subjects. All patients met the clinical diagnostic criteria of ACOS and were confirmed by clinical manifestations, laboratory tests, and imaging examinations. ${ }^{7}$

Patients with organic diseases, immune system diseases, hematological diseases or mental illnesses; those allergic to the medicine used in this study; those unable to cooperate with the treatment; those who had recently 
received hormone therapy and used leukotrienes receptor antagonists, $\beta 2$-agonists, anticholinergic agents within 2 weeks before treatment, were excluded.

All patients were divided into the control group and the observation group using a random number table method, with 43 cases in each group; and received conventional treatment, including control of exercise load, home oxygen therapy or oxygen inhalation. Among them, the control group was treated with inhaled glucocorticoid; that is, budesonide glucocorticoid $100 \mu \mathrm{g}$ per inhalation, 2 times/day. Patients in the observation group were treated with tiotropium bromide on the basis of the control group. Before use, 1 tiotropium bromide capsule $(18 \mu \mathrm{g} /$ capsule) was put into the puncture slot of a special inhaler. The button was pressed with a finger. Both ends of the capsule were punctured with fine needles. The mouthpiece was put deep into the mouth for inhalation. The capsule rotated rapidly with the airflow. The powder in the capsule was ejected and entered the respiratory tract with the airflow. The dosage used was one capsule once a day. Both groups of patients were treated for six months.

The asthma control test (ACT) score and the chronic obstructive pulmonary disease assessment test (CAT) score were compared between the two groups before and after treatment. ACT score and CAT score were conducted by face-to-face interviews between doctors and patients. The total ACT score was 25 points. The higher the score, the less serious the patient's condition was. The total CAT score was 40 points. The higher the score, the more serious the patient's condition was.

Before and after treatment, $5 \mathrm{~mL}$ of fasting venous blood was collected from patients in a state of quietness, and the levels of serum high-sensitivity $\mathrm{C}$-reactive protein (hs-CRP) and IL-6 were detected by enzyme-linked immunosorbent assay. The incidence of adverse reactions during the treatment was observed.

Data was analysed in SPSS version 21. Mean value \pm SD was calculated for numericals like ACT score, CAT score, levels of serum hs-CRP and IL-6, examined by independent sample t-test. Frequencies and percentages were calculated for categorical variables like gender, history of smoking and incidence of adverse reactions. Chi-square test was applied to compare the categorical variables in two groups. Results were considered significant at $p<0.05$.

\section{RESULTS}

Among the 86 patients, 50 were males $(58.14 \%)$ and 36 were females (41.86\%); aged $42-74$ years, mean age $63.71 \pm 2.45$ years. The duration of disease ranged from 6-28 months with mean duration $14.53 \pm 3.02$ months. Patients with a history of smoking were 58 (67.44\%). The number of patients with acute exacerbation in 2 years was 1-5 $(3.4 \pm 1.5)$ times.

Before treatment, there was no significant difference in ACT score and CAT score between the two groups $(P=0.808,0.947$, respectively). After treatment, ACT score was higher in the observation group than that in the control group $(p<0.001)$, and CAT score was lower than that in the control group $(p<0.001$, Table I).

Before treatment, there was no significant difference in serum hs-CRP and IL- 6 levels between the two groups $(p=0.872$ and 0.921 , respectively). After treatment, serum levels of hs-CRP and IL- 6 were lower in the observation group than those in the control group (both $p<0.001$, Table II).

The incidence of adverse reactions, such as dry mouth, heart palpitations, hoarseness, dysuria, and oral candidiasis

Table I: Comparison of ACT and CAT scores before and after treatment.

\begin{tabular}{l|l|c|c}
\hline Index & Time & Control group $(n=43)$ & Observation group $(\mathrm{n}=43)$ \\
\hline ACT score & Before treatment & $10.34 \pm 1.09$ & $10.26 \pm 1.86$ \\
& After treatment & $17.95 \pm 0.81$ & $22.18 \pm 1.24$ \\
\hline CAT score & Before treatment & $26.45 \pm 3.46$ & $26.51 \pm 4.81$ \\
& After treatment & $21.02 \pm 3.70$ & $17.14 \pm 4.62$ \\
\hline
\end{tabular}

Table II: Comparison of serum hs-CRP and IL-6 levels before and after treatment.

\begin{tabular}{l|l|c|c}
\hline Index & Time & Control group $(\mathrm{n}=43)$ & Observation group $(\mathrm{n}=43)$ \\
\hline hs-CRP $(\mathrm{mg} / \mathrm{L})$ & Before treatment & $11.45 \pm 1.86$ & $11.53 \pm 2.67$ \\
& After treatment & $5.84 \pm 0.65$ & $3.22 \pm 1.31$ \\
\hline $\mathrm{IL}-6(\mathrm{ng} / \mathrm{mL})$ & Before treatment & $38.73 \pm 4.84$ & $38.85 \pm 6.17$ \\
& After treatment & $19.24 \pm 2.31$ & 0.872 \\
\hline
\end{tabular}

Table III: Comparison of adverse reactions.

\begin{tabular}{|c|c|c|c|c|c|c|c|c|}
\hline Group & $\mathrm{n}$ & $\begin{array}{l}\text { Dry mouth } \\
{[\mathrm{n}(\%)]}\end{array}$ & $\begin{array}{c}\text { Heart palpitations } \\
{[\mathrm{n}(\%)]}\end{array}$ & $\begin{array}{c}\text { Hoarseness } \\
{[\mathrm{n}(\%)]}\end{array}$ & $\begin{array}{r}\text { Dysuria } \\
{[\mathrm{n}(\%)]}\end{array}$ & $\begin{array}{l}\text { Oral candidiasis } \\
{[n(\%)]}\end{array}$ & $\begin{array}{c}\text { Incidence of } \\
\text { adverse reactions } \\
{[\mathrm{n}(\%)]}\end{array}$ & $p$-value \\
\hline Control group & 43 & $6(13.95)$ & $2(4.65)$ & $2(4.65)$ & $3(6.98)$ & $4(9.30)$ & $17(39.53)$ & 0.033 \\
\hline Observation group & 43 & $2(4.65)$ & $1(2.33)$ & $2(4.65)$ & $2(4.65)$ & $1(2.33)$ & $8(18.60)$ & \\
\hline
\end{tabular}


in the observation group was $18.60 \%$ ( 8 cases), which was lower than that in the control group $(39.53 \%$, 17 cases). The difference was statistically significant $(p=0.033$, Table III).

\section{DISCUSSION}

Glucocorticoids suppress immune responses and prevent inflammation, virus and shock, and are widely used in the treatment of asthma because of its strong anti-inflammatory and anti-allergic effects. ${ }^{8}$ Budesonide is a typical representative of this class of medicines. With better local anti-inflammatory effect, it can effectively improve the patient's clinical symptoms and lung function, as well as inhibit the patient's airway inflammation.9,10 Tiotropium bromide is a highly effective, long-acting anticholinergic drug that selectively acts on patients' M1 and M2 receptors. It has a longlasting and mild effect with a low incidence of adverse reactions. It can not only effectively alleviate the clinical response of asthma-COPD patients during the disease, but also improve the patient's lung function and expand bronchial smooth muscle.11,12

This study showed that after treatment, ACT score of the observation group was higher than that of the control group, and CAT score was lower than that of the control group. It suggested that inhaled glucocorticoid combined with tiotropium bromide can effectively relieve the clinical symptoms of ACOS patients and help control the development of the disease. It also showed that the incidence of adverse reactions was lower in the observation group than that in the control group, suggesting that inhaled glucocorticoid combined with tiotropium bromide in the treatment of ACOS has relatively high safety. This general conclusion is consistent with previous research suggesting that tiotropium bromide is a safe and effective treatment choice for moderate-to-severe COPD. 13

The hs-CRP is an acute phase protein synthesised by liver cells when the body is subjected to inflammatory stimuli such as microbial invasion or tissue damage, and it is not affected by radiotherapy, chemotherapy, and corticosteroid treatment. ${ }^{14}$ When the inflammatory reaction occurs in the respiratory tract, hs-CRP levels can indirectly reflect the degree of airflow limitation in the patient. ${ }^{15,16}$ Mainly secreted by monocytes, IL-6 can promote the aggregation and activation of inflammatory cells, the expression of adhesion molecules, and participate in the inflammatory reaction. ${ }^{17}$ Studies have shown that the pathogenic mechanism of ACOS may be related to inflammatory reactions in the body. The combined action of a variety of inflammatory cells and inflammatory mediators (hs-CRP, IL-6, etc.) causes changes in the permeability of bronchial epithelial cells, resulting in cell dysfunction, even apoptosis and destruction of extracellular matrix. The change in airway smooth muscle function leads to airway remodelling and airflow obstruction, eventually resulting in increased pulmonary vascular resistance and severely impaired lung function. ${ }^{18,19}$ Barrecheguren et al. have shown that IL-6 can mediate inflammation and cause irreversible airway remodelling in ACOS patients, and its increased content can promote the body to generate large amounts of reactive oxygen, causing indirect or direct injury to lung tissue. 20

In the present study, serum levels of hs-CRP and IL-6 were lower in the observation group than those in the control group after treatment. This can effectively prove that the combination of inhaled glucocorticoid and tiotropium bromide in the treatment of ACOS patients can significantly reduce the level of serum inflammatory factors, relieve the inflammatory response in vivo, and promote the favourable outcome of the disease. The combination of inhaled glucocorticoid and tiotropium bromide exerts synergistic effects in a variety of mechanisms and has a good therapeutic effect on clinical treatment of ACOS patients.

There are some limitations of this study. First, the sample size is relatively small. Second, the severity of the disease in subjects are not grouped and only mentioned on the basis of symptoms score as mean values. Thirdly, there is no comparison between the two groups in lung functions or quality of life assessment, 6-minute walk test, etc. These limitations can be studied further in future.

\section{CONCLUSION}

Compared with inhaled glucocorticoid, inhaled glucocorticoid combined with tiotropium bromide treatment can more effectively reduce the serum levels of hs-CRP and IL-6, which is beneficial to control the development of ACOS.

\section{REFERENCES}

1. Milkowska-Dymanowska J, Bialas AJ, Górski P. Selected aspects of medical care for patients with asthma and chronic obstructive pulmonary disease (COPD) in Poland. Adv Respir Med 2017; 85:179-85.

2. Cadeddu C, Capizzi S, Colombo D, Nica M, De Belvis AG. Literature review of gender differences in respiratory conditions: A focus on asthma and chronic obstructive pulmonary disease (COPD). Ig Sanita Pubb/ 2016; 72:481-504.

3. Barrecheguren M, Esquinas C, Miravitlles M. The asthmachronic obstructive pulmonary disease overlap syndrome (ACOS): Opportunities and challenges. Curr Opin Pulm Med 2015; 21:74-9.

4. Kankaanranta H, Harju T, Kilpeläinen M, Mazur W, Lehto JT, Katajisto $\mathrm{M}$, et al. Diagnosis and pharmacotherapy of stable chronic obstructive pulmonary disease: the finnish guidelines. Basic Clin Pharmacol Toxicol 2015; 116:291-307.

5. Zhou L, Song LQ, Cheng XD, Niu PX, Wang X. Clinical efficacy of inhaled corticosteroids in the treatment of patients with 
acos and its influence on the endothelial cell function. Prog Modern Biomed 2017; 5892-5.

6. Mamary AJ, Criner GJ. Tiotropium bromide for chronic obstructive pulmonary disease. Exp Rev Respir Med 2009; 3:211-20.

7. Hardin M, Cho M, McDonald ML, Beaty T, Ramsdell J, Bhatt S, et al. The clinical and genetic features of COPD-asthma overlap syndrome. Eur Respir J 2014; 44:341-50.

8. Corrigan CJ, Brown PH, Barnes NC, Tsai JJ, Frew AJ, Kay AB. Glucocorticoid resistance in chronic asthma. Peripheral blood T-lymphocyte activation and comparison of the T-lymphocyte inhibitory effects of glucocorticoids and cyclosporin A. Am Rev Respir Dis 1991; 144:1026-32.

9. Li B, Han S, Liu F, Kang L, Xv C. Budesonide nebulization in the treatment of neonatal ventilator associated pneumonia. Pak J Med Sci 2017; 33:997-1001.

10. Belchamber KB, Thomas CM, Dunne AE, Barnes PJ, Donnelly LE. Comparison of fluticasone propionate and budesonide on COPD macrophage and neutrophil function. Int $J$ Chron Obstruct Pulmon Dis 2018; 13:2883-97.

11. Gregory JA, Kemi C, Ji J, Karén J, Delin I, Gerde P, et al. Effects of tiotropium bromide on airway hyper-responsiveness and inflammation in mice exposed to organic dust. Pulm Pharmacol Ther 2017; 48:203-10.

12. Ohbayashi $H$. Comparison of the rapid effects of single inhalations of formoterol and tiotropium bromide on respiratory function and COPD symptoms in a randomized crossover study. Respir Investig 2017; 55:348-56.

13. Alcibey AG, Isabel A. Tiotropium bromide in chronic obstructive pulmonary disease and bronchial asthma. J Clin Med Res 2015; 7: 831-9.
14. Pfützner A, Schöndorf T, Hanefeld M, Forst T. High-sensitivity c-reactive protein predicts cardiovascular risk in diabetic and nondiabetic patients: effects of insulin-sensitizing treatment with pioglitazone. J Diabetes Sci Technol 2010; 4: 706-16.

15. Gläser S, Ittermann T, Koch B, Völzke H, Wallaschofski $H$, Nauck $M$, et al. Airflow limitation, lung volumes and systemic inflammation in a general population. Eur Respir J 2012; 39: 29-37.

16. Huang J, Chen X, Wang ZK. Combined application value analysis of detecting serum levels of pct and hs-crp in children with acute respiratory tract infection. J Clin Exp Med 2013; 12:1645-6.

17. Khyani IAM, Qureshi MA, Mirza T, Farooq MU. Detection of interleukins- 6 and 8 in saliva as potential biomarkers of oral pre-malignant lesion and oral carcinoma: A breakthrough in salivary diagnostics in Pakistan. Pak J Pharm Sci 2017; 30: 817-23.

18. Kodama Y, Kishimoto Y, Muramatsu Y, Tatebe J, Yamamoto Y, Hirota $\mathrm{N}$, et al. Antioxidant nutrients in plasma of Japanese patients with chronic obstructive pulmonary disease, asthmaCOPD overlap syndrome and bronchial asthma. Clin Respir J 2017; 11:915-24

19. Fu JJ, Mcdonald VM, Gibson PG, Simpson JL. Systemic inflammation in older adults with asthma-copd overlap syndrome. Allergy Asthma Immunol Res 2014; 6:316-24.

20. Barrecheguren $M$, Románrodríguez $M$, Miravitlles $M$. Is a previous diagnosis of asthma a reliable criterion for asthmaCOPD overlap syndrome in a patient with COPD? Int J Chron Obstruct Pulmon Dis 2015; 10:1745-52. 